\title{
Coumarin-modified microporous-mesoporous Zn-MOF-74 showing ultra-high uptake capacity and photo-switched storage/release of $\mathrm{U}^{\mathrm{VI}}$ ions
}

Le Zhang, Lin Lin Wang, Le Le Gong, Xue Feng Feng,Ming Biao Luo, and Feng Luo*

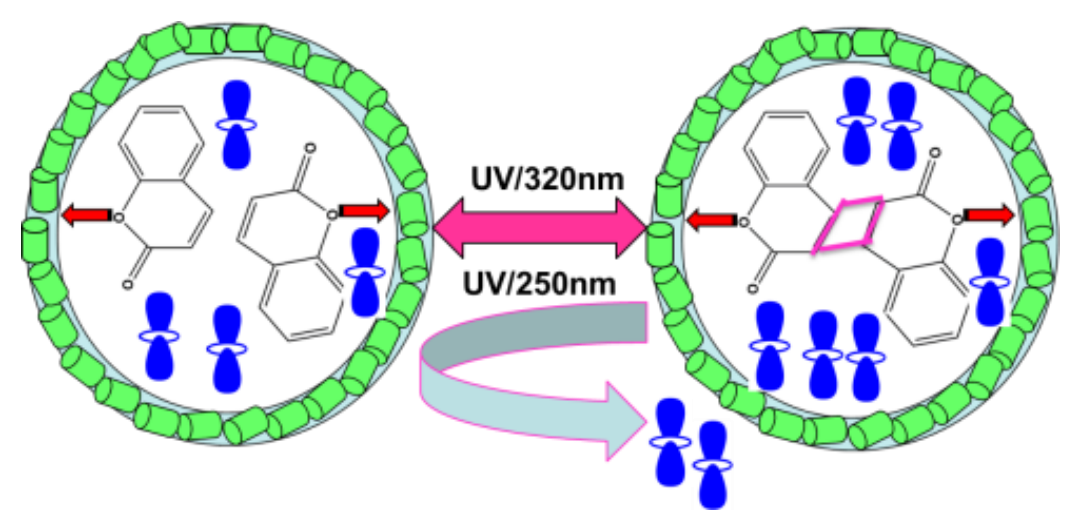

Herein, through coordination-based post-synthetic strategy, microporous-mesoporous Zn-MOF-74 was easily functionalized by grafting coumarin on coordinatively unsaturated $\mathrm{Zn}$ (II) centers, yielding a series of coumarin-modified Zn-MOF-74 materials. The obtained samples afforded ultra-high adsorption capacity for $\mathbf{U}^{\mathrm{VI}}$ ions from water with maximum adsorption capacities as high as $360 \mathrm{mg} / \mathrm{g}$ (the record value in MOFs) and remarkable photo-switched capability of $50 \mathrm{mg} / \mathrm{g}$. 


\title{
Coumarin-modified microporous-mesoporous Zn-MOF-74 showing ultra-high
} uptake capacity and photo-switched storage/release of $U^{\mathrm{VI}}$ ions

\author{
Le Zhang, Lin Lin Wang, Le Le Gong, Xue Feng Feng, Ming Biao Luo, and Feng Luo*
}

State Key Laboratory for Nuclear Resources and Environment, and School of Biology, Chemistry and Material Science, East China University of Technology, Nanchang, Jiangxi 344000, China, ecitluofeng@163.com

Abstract: Driven by an energy crisis but consequently puzzled by various environmental problems, uranium, as the basic material of nuclear energy, is now receiving extensive attentions. In contrast to numerous sorbents applied in this field, metal-organic framework (MOFs), as a renovated material platform, has only recently been developed. How to improve the adsorption capacity of MOF materials towards $\mathrm{U}^{\mathrm{VI}}$ ions, as well as taking advantage of the nature of these MOFs to design photo-switched behaviour for photo-triggered storage/release of $\mathrm{U}^{\mathrm{VI}}$ ions are at present urgent problems and great challenges to be solved. Herein, we show a simple and facile method to target the goal. Through coordination-based post-synthetic strategy, microporousmesoporous Zn-MOF-74 was easily functionalized by grafting coumarin on coordinatively unsaturated $\mathrm{Zn}$ (II) centers, yielding a series of coumarin-modified Zn-MOF-74 materials. The obtained samples displayed ultra-high adsorption capacity for $\mathrm{U}^{\mathrm{VI}}$ ions from water at $\mathrm{pH}$ value of 4 with maximum adsorption capacities as high as $360 \mathrm{mg} / \mathrm{g}$ (the record value in MOFs) and a remarkable photo-switched capability of $50 \mathrm{mg} / \mathrm{g}$ at $\mathrm{pH}$ value of 4 . To the best of knowledge, and in contrast to the well-known photo-switched behaviour towards $\mathrm{CO}_{2}$, dye (propidium iodide), as well as fluorescence observed in MOFs, this is the first study that shows a photo-switched behaviour towards radioactive $\mathrm{U}^{\mathrm{VI}}$ ions in aqueous solution.

Keywords: MOF; $\mathrm{U}^{\mathrm{VI}}$ uptake; Photo-switching behaviour; Coumarin; Hybrid material

\section{Introduction}


As predicated by the International Atomic Energy Agency, nuclear power as a mature technology is now receiving major attentions during this century. This is mainly because of the rapidly growing energy demand and concerns regarding climate change due to carbon sources, as well as the decommissioning of more than 400 reactors around the world, built between 1970-1980. ${ }^{1}$ Corresponding to this increasing demand, one serious concern about potential risks from radiation sources is the toxic waste generated into the environment, natural water resources, and its effect on human health. ${ }^{2-3}$ Typically, $\mathrm{U}^{\mathrm{VI}}$ uranium are the major source of nuclear power, thus high-performance and safe storage/release of uranium are of urgent need, great scientific and practical significance, not only in removal of uranium-containing waste for protection of both human health and ecological security, but also in reutilization of uranium resources for sustainable development of nuclear energy.

For this purpose, many efforts, including liquid-liquid extraction, ${ }^{4}$ ion-exchange/absorption, ${ }^{5}$ adsorption, ${ }^{6}$ and chemical/biochemical reductive precipitation, ${ }^{7}$ have been devoted. Among these methods, the most common method in the industry currently to extract U(VI) from aqueous solutions is solvent extraction such as tributylphosphines and tertiary amines, ${ }^{8-9}$ due to its unique merit such as low cost, fast speed, high efficiency, easy to automatic control, and repeated operation. But this is somewhat restricted in practice by the thermostalibty of extractant. On the other hand, porous sorbent based adsorption approach is one of the most convenient methods for $\mathrm{U}^{\mathrm{VI}}$ treatment. First the category of sorbents covers a large range of materials that included activated carbon, ${ }^{10}$ activated carbon-silica aerogel composite materials, ${ }^{11}$ barium titanate, ${ }^{12}$ gallocyanine grafted hydro-gel, ${ }^{13}$ ion-imprinted polymers, ${ }^{14}$ polyphenolic compounds, ${ }^{15}$ as well as recently developed layered sulfide ${ }^{16-17}$ and metal-organic frameworks (MOFs) ${ }^{18-20}$. Secondly the operation to carry out uranium adsorption/release, based on porous sorbents, is often facile. Among them, MOFs, as a renovated porous platform, has only recently been developed for such purposes. Especially, numerous unique merits such as high BET, uniform pore, functionalized pore wall, and structural 
diversity make this material more attractive. Moderate chemostability and thermostability of the MOF skeleton in conjunction with their preferred chemical adsorption towards $\mathrm{U}^{\mathrm{VI}}$ ions largely benefit the applications in storage and release of uranium in practice. Nevertheless, one serious concern that should be noticed is that the low-cost material available at present in this category is still scarce. Also, seeking for general approach to enhance $\mathrm{U}^{\mathrm{VI}}$ adsorption capability of MOFs, especially simple MOFs, is of urgent need but still remains a big challenge. Moreover, attempts of exploiting some photo-sensitive MOFs for photo-switched storage and release of $\mathrm{U}^{\mathrm{VI}}$ are also special of interest in the green storage/release of $\mathrm{U}^{\mathrm{VI}}$.

MOF-74 material is one of the most economical MOFs (such as Zn-MOF-74, cost $<2$ $\$ / \mathrm{kg}){ }^{21}$ showing a robust framework, oxygen-rich interior pore, and potential coordination-unsaturated sites, is now extensively applied in many research fields like magnetism, catalysis, separation, batteries, pharmaceutics, and so on. ${ }^{22}$ The facile preparation of microporous-mesoporous presented by Yue and co-workers is one that deserver merit. ${ }^{23}$ In this work, as described in Scheme 1, the adsorption of $\mathrm{U}^{\mathrm{VI}}$ ions on crystal samples of Zn-MOF-74 (A-1), its microporous-mesoporous samples (A-2), and its coumarin loading samples (A-3), was systematically studied. The motive of this research is focused on how to enhance the $\mathrm{U}^{\mathrm{VI}}$ adsorption capability upon MOFs materials, and construct photo-sensitive MOFs for photo-switched storage/release of $\mathrm{U}^{\mathrm{VI}}$.

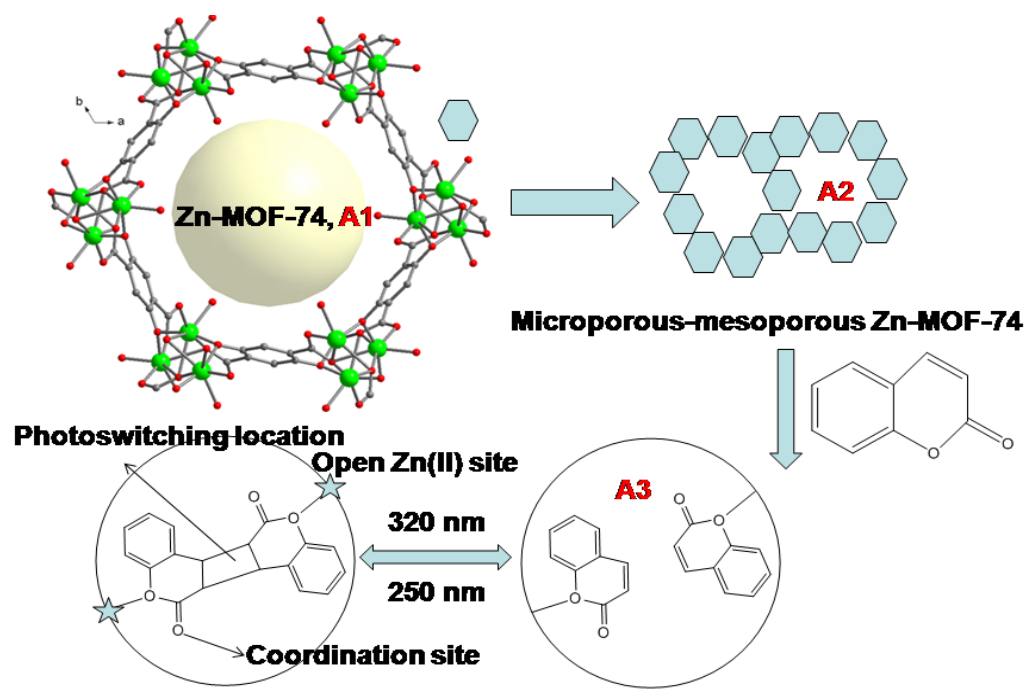


Scheme 1. The materials used in this work for adsorption and photo-switching storage/release of $\mathrm{U}^{\mathrm{VI}}$ ions.

\section{Experimental detail}

\subsection{Synthesis.}

2.1.1 Microporous Zn-MOF-74. A DMF/isopropanol/ $\mathrm{H}_{2} \mathrm{O}$ (2:0.1:0.1, volume ratio) of $\mathrm{Zn}\left(\mathrm{NO}_{3}\right)_{2} \cdot 6 \mathrm{H}_{2} \mathrm{O}(0.1 \mathrm{mmol}), 2,5$-dihydroxy-1,4-benzenedicarboxylic acid $(0.05 \mathrm{mmol})$ was sealed in a Teflon reactor, heated at $110^{\circ} \mathrm{C}$ for 3 days, and cooled to room temperature at $3^{\circ} \mathrm{C} / \mathrm{h}$. Subsequently, block crystals were yielded and dried naturally in air for three days to obtain a product with $85 \%$ yield based on $\mathrm{Zn}$. The resulted crystals were washed first with $20 \mathrm{~mL}$ of DMF and then $20 \mathrm{~mL}$ of methanol. The solid was kept immersed in methanol for 3 days with fresh solvent changed for the same amount and repeated three times. They were then degassed in Belsorp-max under vacuum at $180^{\circ} \mathrm{C}$ for $24 \mathrm{~h}$. Element analysis for the activated samples $(71 \%$ yield based on Zn): calc. C/29.57, H/0.62; exp. C/29.16, H/0.66.

2.1.2 Microporous-mesoporous $\mathrm{Zn-MOF-74.} \mathrm{The} \mathrm{clear} \mathrm{solution} \mathrm{of} \mathrm{Zn}\left(\mathrm{CH}_{3} \mathrm{COO}\right)_{2} \cdot 2 \mathrm{H}_{2} \mathrm{O}(0.343 \mathrm{~g})$ in $20 \mathrm{~mL}$ DMF was added dropwise to the clear solution of 2,5-dihydroxy-1,4-benzenedicarboxylic acid $(0.099 \mathrm{~g})$ in $20 \mathrm{~mL}$ DMF, immediately leading to a precipitate. Then this was further stirred for $15 \mathrm{~min}$, and the solid was recovered by centrifugation and washed first with $20 \mathrm{~mL}$ of DMF and then $20 \mathrm{~mL}$ of methanol. The solid was kept immersed in methanol for 3 days with fresh solvent changed for the same amount and repeated three times. They were then degassed in Belsorp-max under vacuum at $200^{\circ} \mathrm{C}$ for $24 \mathrm{~h}$. Element analysis for the activated samples $(76 \%$ yield based on Zn)): calc. C/29.57, H/0.62; exp. C/29.79, H/0.53.

2.1.3 Coumarin-modified microporous-mesoporous Zn-MOF-74. 0.5 g activated microporousmesoporous Zn-MOF-74 was suspended in a solution containing 20ml of n-hexane and $0.07 \mathrm{~g}$ (12 $\mathrm{wt} \%) / \mathrm{or} 0.11 \mathrm{~g}(22 \mathrm{wt} \%) /$ or $0.22 \mathrm{~g}(30 \mathrm{wt} \%) /$ or $0.30 \mathrm{~g}$ (37 wt $\%)$ of coumarin under stirring at ambient temperature for $15 \mathrm{~min}$. $\mathrm{n}$-Hexane was evaporated by a rotary evaporator at $333 \mathrm{~K}$ for $2 \mathrm{~h}$, 
and the resulting material was washed by ethanol $(30 \mathrm{~mL})$ three times and then dried under vacuum at $373 \mathrm{~K}$ for $24 \mathrm{~h}$.

2.2 Materials and General Methods. Reagents and solvents were commercially available (Alfa) and were used without further purification. Thermogravimetric analysis (TGA) was performed by a TGA Q500 thermal analysis system. Data were analyzed using the TA Universal Analysis software package. X-ray powder diffraction were collected by a Bruker AXS D8 Discover powder diffractometer at $40 \mathrm{kV}, 40 \mathrm{~mA}$ for $\mathrm{Cu} \mathrm{K \alpha},(\lambda=1.5406 \AA)$. The simulated powder patterns were calculated by Mercury 1.4. The purity of the bulk products were determined by comparison of the simulated and experimental PXRD patterns. Infrared Spectra (IR) were measured by a Bruker VERTEX 70 spectrometer in the $500-4000 \mathrm{~cm}^{-1}$ region. The gas sorption isotherms were collected on a Belsorp-max. Ultrahigh-purity-grade (>99.999\%) $\mathrm{N}_{2}, \mathrm{CO}_{2}$ gases were used during the adsorption measurement. To maintain the experimental temperatures, liquid nitrogen $(77 \mathrm{~K})$ and temperature-programmed water bath $(298 \mathrm{~K})$ were used respectively. SEM and EDS measurements were carried out using a Hitachi S-4800 microscope.

2.3 Uranium Uptake Experiments. The uranium uptake was carried out via a batch method. For example, uptake experiments were performed at controlled $\mathrm{pH}$ values of 1-7 and $298 \mathrm{~K}$ by adding $10 \mathrm{mg}$ MOF material to $10 \mathrm{~mL}$ of a uranyl solution in a $50 \mathrm{~mL}$ high-density Polymer polypropylene tube (previously cleaned with $5 \% \mathrm{HNO}_{3}$ and rinsed three times with ultrapure water). The solution $\mathrm{pH}$ was adjusted to the desired value of 1-7 by $\mathrm{HCl}(1 \mathrm{M}) / \mathrm{NH}_{3} \mathrm{H}_{2} \mathrm{O}(1: 1)$ and determined by $\mathrm{pH}$ meter. After shaking for a predesigned time $(0-5 \mathrm{~h})$ with fixed temperature in the ZWY-240 in a constant shaking incubator, the suspension was centrifuged at $10,000 \mathrm{rpm}$ for $10 \mathrm{~min}$, then the sorbent was separated by centrifugation and the rest solution is analyzed for $U$ concentration by a spectrophotometer (TU-1901), and further confirmed by inductively coupled plasma-mass spectroscopy (ICP-MS, NexION 300X). Arsenazo III dye was used as the color-developing agent for U, 
giving an intense absorption band at $650 \mathrm{~nm}$. Before the determination, the solution was diluted 2.5-100 times to make sure that the finally uranium concentration meets the standard curve in the range of $0.1-3 \mathrm{mg} / \mathrm{L}$.

\section{Results and discussion}

The crystal samples A-1 was synthesized by the reaction of 2,5-dihydroxy-1,4-benzenedicarboxylic acid and $\mathrm{Zn}\left(\mathrm{NO}_{3}\right)_{2} \cdot 6 \mathrm{H}_{2} \mathrm{O}$ in the ratio of 2:1 in mixed solvents of DMF/isopropanol/ $\mathrm{H}_{2} \mathrm{O}$ (2:0.1:0.1, volume ratio). The structure was determined by single crystal X-ray diffraction and agrees well with literature reports. ${ }^{22}$ The phase purity of the bulk samples were confirmed by powder X-ray diffraction (PXRD, Figure 1). According to the synthesis method in the literature, ${ }^{23} \mathbf{A - 2}$ is synthesized by stirring 2,5-dihydroxy-1,4-benzenedicarboxylic acid and $\mathrm{Zn}\left(\mathrm{CH}_{3} \mathrm{COO}\right)_{2} \cdot 2 \mathrm{H}_{2} \mathrm{O}$ in DMF for $15 \mathrm{~min}$ at room temperature. The phase purity of $\mathbf{A - 2}$ was further confirmed by PXRD through comparing PXRD patterns of A-2 with PXRD patterns of A-1. The preparation of A-3 was also referred to the literature method by loading coumarin in activated A-2. ${ }^{24}$ The comparison of PXRD patterns between A-2 and its coumarin-modified phase A-3 suggests that the special MOF skeleton of Zn-MOF-74 was well maintained even after loading coumarin inside it.

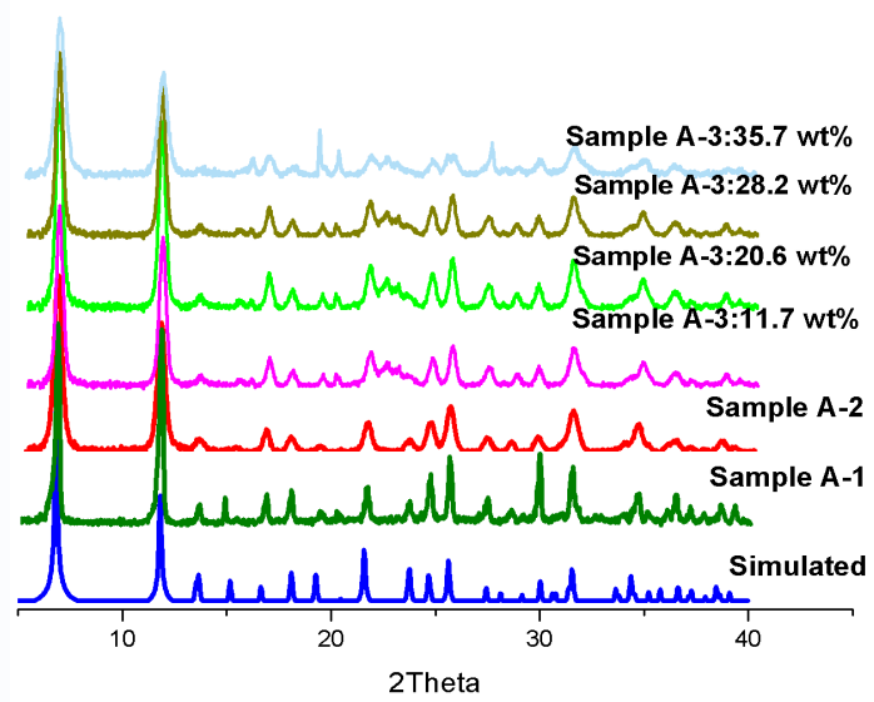


Figure 1. PXRD patterns simulated from single crystal data of Zn-MOF-74, and PXRD patterns of samples A-1, A-2, and A-3 series with different loads of coumarin.
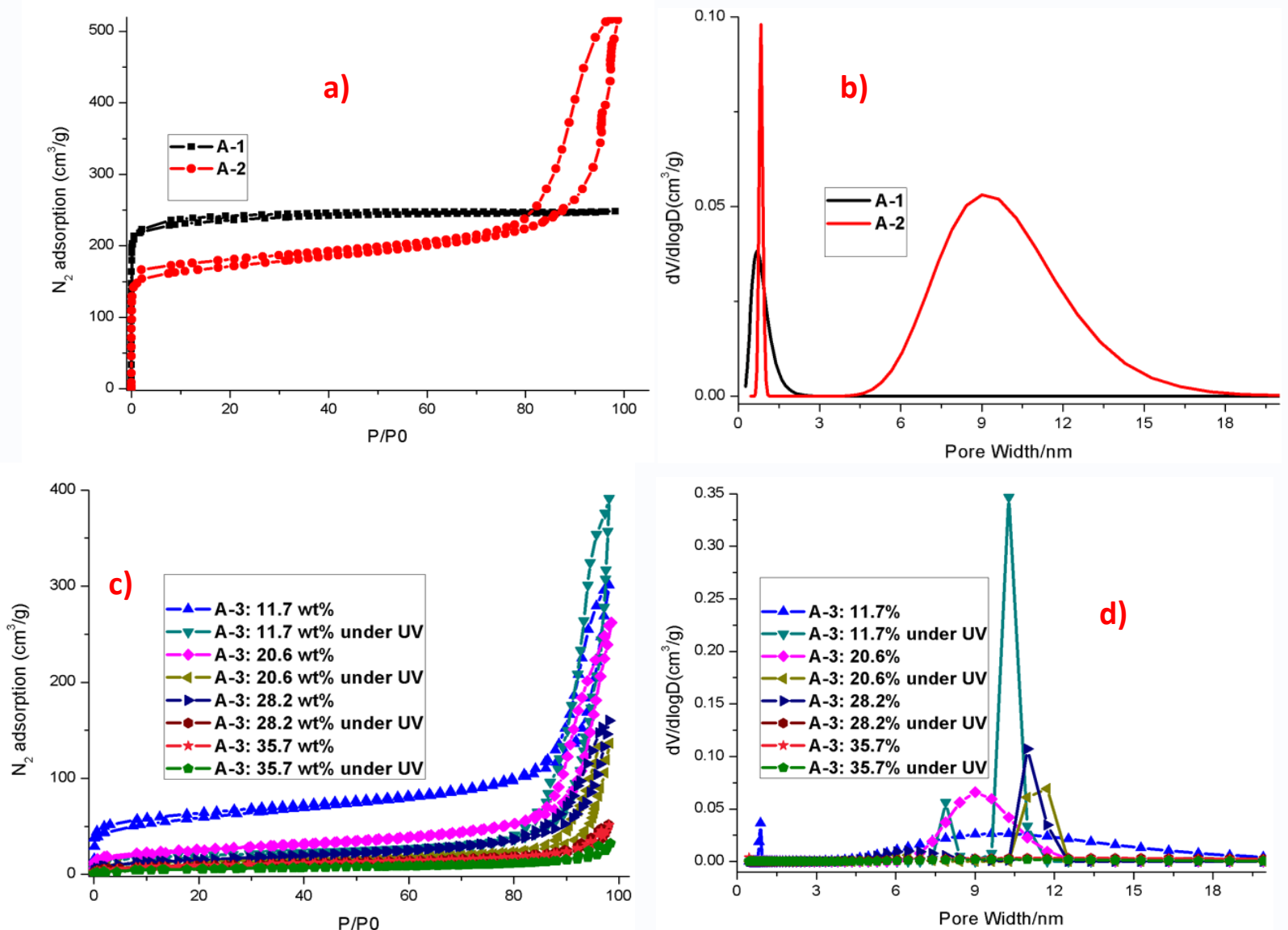

Figure 2. a), c) $\mathrm{N}_{2}$ adsorption/desorption isotherms at $77 \mathrm{~K}$ of $\mathbf{A - 1}, \mathbf{A}-\mathbf{2}$, and $\mathbf{A}-\mathbf{3}$ series; b), d) their corresponding pore size distributions (PSDs) calculated by NLDFT. Note that, for example, 'A-3:11.7wt\%' means that in this sample about $11.7 \mathrm{wt} \%$ coumarin was loaded inside A-3, while 'A-3:11.7wt\% under UV' means that before carrying out $\mathrm{N}_{2}$ adsorption experiment the A-3:11.7wt\% sample were irradiated under UV $(320 \mathrm{~nm})$ for $30 \mathrm{~min}$.

As shown in Figure 2, the aperture of samples A-1 estimated by $\mathrm{N}_{2}$ adsorption at $77 \mathrm{~K}$, is ca. $0.79 \mathrm{~nm}$, which is consistent with the value reported in the literature. ${ }^{22}$ In contrast to the type-I isotherm for microporous samples $\mathbf{A - 1}$, the $\mathrm{N}_{2}$ adsorption-desorption isotherm of samples A-2 displays type-IV isotherms with hysteresis loops characteristic of large constricted mesopores. The pore-size distribution (PSD), calculated by nonlocal density functional theory (NLDFT) suggests these are microporous-mesoporous materials with the distributions of a micropore around $0.84 \mathrm{~nm}$ 
and a mesopore width as large as of $9 \mathrm{~nm}$. This is comparable with that reported recently by Yue $e t$ $a l,{ }^{23}$ as for similar structures, the $\mathbf{A - 3}$ series, the micropores were found to gradually disappear along with increasing loading amount of coumarin (for example, more than 20.6wt\%). Correspondingly, the widepore size distribution observed in samples A-2 becomes narrow in pore size distribution in the $\mathbf{A - 3}$ series. There results, combined with the very low $\mathrm{CO}_{2}$ adsorption in $\mathbf{A - 3}$ : 11.7wt\%, strongly suggests that both micropores and mesopores were modified by coumarin.

The loading amount of coumarin is estimated by both EA (element analysis) and thermogravimetric analyses (TG). The results in detail are listed in Table S1 and Figure S1. And the A-3 series were named as A-3: 11.7wt\%, A-3: 20.6wt\%, A-3: 28.2wt\%, A-3: 35.7wt\%, respectively, where for example " $11.7 \mathrm{wt} \%$ " means that the loading amount of coumarin on MOF material is about $11.7 \mathrm{wt} \%$. The amount of $\mathrm{CO}_{2}$ adsorption at $298 \mathrm{~K}$ and $100 \mathrm{kPa}$ is $120.2 \mathrm{~cm}^{3} / \mathrm{g}$ for A-2, typical of $\mathrm{CO}_{2}$ adsorption capability for $\mathrm{Zn-MOF-74} \mathrm{showing} \mathrm{open} \mathrm{Zn}$ (II) site (Figure S7). However, this is sharply reduced to $12.1 \mathrm{~cm}^{3} / \mathrm{g}$ for $\mathbf{A - 2}$ after loading $11.7 \mathrm{wt} \%$ coumarin, which is likely the results of the prior occupation of coumarin oxygen atom on the open $\mathrm{Zn}$ (II) site (Figure S2).

The ultraviolet (UV) diffuse reflectance spectrum of coumarin-modified samples (A-3 series) is shown in Figure S3. In comparison to samples A-2, new peak is observed in A-3 series around $313 \mathrm{~nm}$, characteristic of absorption bond for coumarin. ${ }^{24}$ Irradiation of sample A-3 series with UV light for 1 min with wavelengths of $320 \mathrm{~nm}$ results in sharp increase in the absorption band around $270 \mathrm{~nm}$, indicative of occurrence of photodimerization inside A-3 series. ${ }^{24}$ This can be further reflected in the $\mathrm{N}_{2}$ adsorption (Figure 2) and TG tests (Figure S4). For example, the amount of $\mathrm{N}_{2}$ adsorption at $\mathrm{P} / \mathrm{P} 0=1$ is $516.7 \mathrm{~cm}^{3} / \mathrm{g}$, whereas after UV (320 nm) irradiation for 30 minutes this decreases to $302.2 \mathrm{~cm}^{3} / \mathrm{g}$, giving a difference of $41.5 \%$ in reduction. However, along with increasing the loading of coumarin, similar trends were also observed but with less alteration (for example, a photo-switching 
alteration of 34.3\% was observed for A-3 after loading 35.7 wt $\%$ coumarin). This strongly suggests that the formation of a more dense phase along with increasing the loading of coumarin occured. Accordingly, we can find that such "open-close double doors" system in A-3 after loading coumarin of less than $20.6 \mathrm{wt} \%$ has little effect on sealing guest molecules. Moreover, the thermostability of A-3 series with or without UV was investigated. Figure S5 presented the observation of alteration for the weight loss before $114^{\circ} \mathrm{C}$ (losing guest molecules) in A-3 series materials with or without UV irradiation. Notably, only samples A-3: 28.2\% and A-3: 35.7\% displayed big difference. For instance, A-3: 28.2wt\% losses its guest molecule of $22.1 \%$ before $114^{\circ} \mathrm{C}$, whereas it after UV irradiation only give a weight loss of $6.9 \%$, giving a large change of $68.7 \%$ and suggesting an effective seal of guest. Even more, a change of $72.5 \%$ is observed for A-3: 35.7wt\% with/without UV irradiation. This agrees with that in this situation such "open-close double doors" system can excellently seal guest molecules. Moreover, to exclude that such photo-switching behaviour may result from the structural transformation after photodimerization inside $\mathbf{A - 3}$ series, the comparison of PXRD patterns between A-3 series and that after UV irradiation was carried out, where the PXRD patterns between them could be well matched, thus strongly suggesting unchanged skeleton between them (Figure S5) and that these MOF materials did not suffer radiation damage from the UV exposure.

To evaluate the ability of A-1, A-2, and A-3 series to adsorb $\mathrm{U}^{\mathrm{VI}}$ ions, batch experiments were carried out. As shown in Figure 3 and Figure S6-11, the adsorption capability of A-1 was 190 mg/g at $\mathrm{pH}=4$ and room temperature. Note that most often uranium containing solutions in the industry are often much more acidic with $\mathrm{pH} \sim 1$, and the optimal $\mathrm{pH}$ of 4 in this work is much higher than often found in the industry, thus, in practice this would cause the precipitation of other ions in solution such as Fe or $\mathrm{Al}$ phases. ${ }^{25-26}$ 
The kinetics of adsorption can be well fitted by the pseudo-second-order rate equation (Figure S7), implying a dominating chemical adsorption rather than physical adsorption. The sorption isotherms observed in this work, according to the Langmuir model (Figure S8), indicate that the adsorption of $\mathrm{U}^{\mathrm{VI}}$ ions is localized in a monolayer. ${ }^{20-22} \mathrm{~A}$ similar situation is observed in A-2, but the adsorption capability of it is largely enhanced up to $300 \mathrm{mg} / \mathrm{g}$. This should benefit from the microporous-mesoporous form in $\mathbf{A - 2}$, relative to the microporous form in A-1. Notably, the adsorption capability can be further enhanced via loading of coumarin inside of A-2. For instance, the material of loading 11.7 wt $\%$ coumarin inside of A-2 could generate adsorption capability as high as of $360 \mathrm{mg} / \mathrm{g}$. This largely exceeds the best values in MOFs such as $\mathrm{UiO}-68-\mathrm{P}(\mathrm{O})(\mathrm{OEt})_{2}(217 \mathrm{mg} / \mathrm{g})^{18}$ and MOF-76 $(298 \mathrm{mg} / \mathrm{g}){ }^{19}$ and is of comparable with the best documented $\mathrm{U}^{\mathrm{VI}}$ sorbents $(307-380 \mathrm{mg} / \mathrm{g})$ like that of KMS-1 (382 mg/g), ${ }^{16} \mathrm{~S}_{\mathrm{x}}-\mathrm{LDH}(\mathrm{x}=2,4,330 \mathrm{mg} / \mathrm{g}),{ }^{17}$ and functionalized hydrothermal carbon (HTC) $(307 \mathrm{mg} / \mathrm{g})^{15}$. However, continuing to increase the amount of coumarin inside of A-2 results in the decrease of $\mathrm{U}^{\mathrm{VI}}$ adsorption capability, as observed in A-3: 20.6wt\% (356 $\mathrm{mg} / \mathrm{g})$, A-3: $28.2 \mathrm{wt} \%(350 \mathrm{mg} / \mathrm{g})$, and A-3: $35.7 \mathrm{wt} \%(280 \mathrm{mg} / \mathrm{g})$, as in this situation, although increasing the amount of coumarin created more chance to chemically adsorb $\mathrm{U}^{\mathrm{VI}}$ ions, however, the finally $\mathrm{U}^{\mathrm{VI}}$ adsorption capability is seriously restricted by the gradual decrease of effective pore like observed in Figure $2 c$. The small variety in $\mathrm{U}^{\mathrm{VI}}$ adsorption capacity between A-3: 20.6wt \% and A-3: 28.2wt \% is mainly due to that such increase of coumarin on MOF material that would create more chemical adsorption sites for $\mathrm{U}^{\mathrm{VI}}$ ions could compensate the decrease of effective pore (due to the increase of coumarin on MOF material) that would reduce $\mathrm{U}^{\mathrm{VI}}$ adsorption capacity. By contrast, when loading of $35.7 \mathrm{wt} \%$ coumarin on MOF materials would largely reduce the effective pore for $\mathrm{U}^{\mathrm{VI}}$ adsorption, although more chemical adsorption sites for $\mathrm{U}^{\mathrm{VI}}$ ions are created after loading of more coumarin on MOF materials. And in this situation, the balance is largely destroyed, thus 
generating a bigger variety in $\mathrm{U}^{\mathrm{VI}}$ adsorption capacity between $\mathbf{A - 3}$ : 20.6wt\% and A-3: $35.7 \mathrm{wt} \%$.

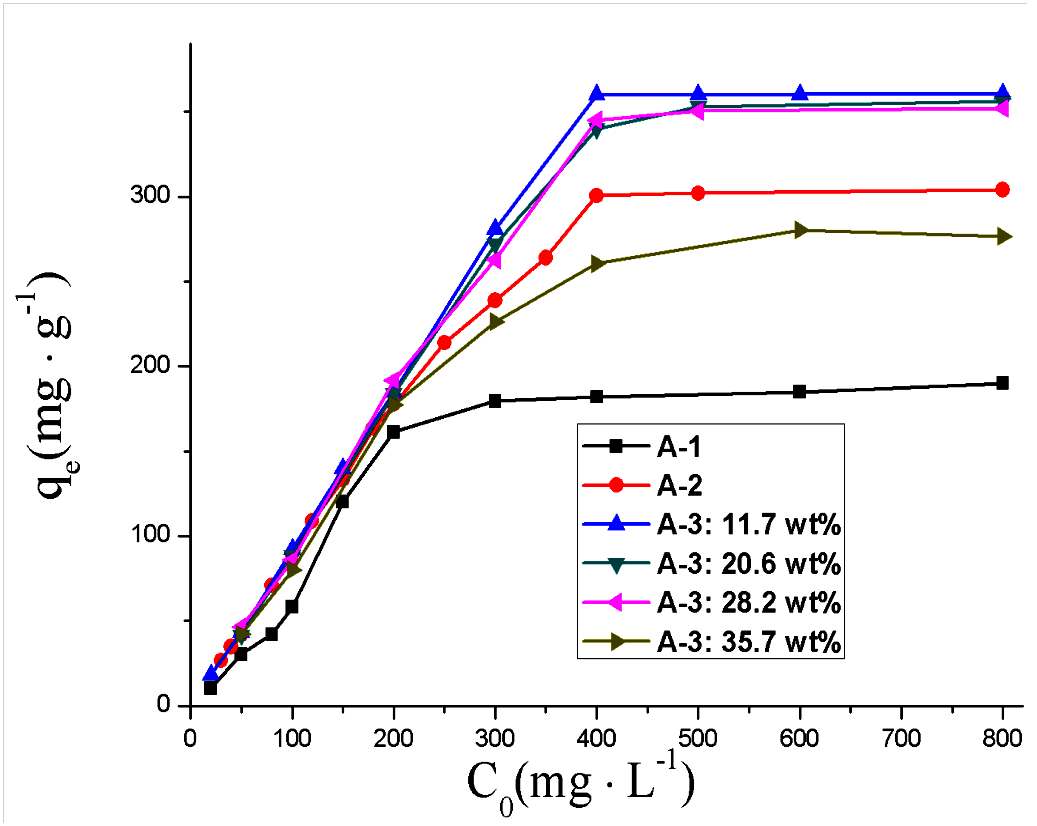

Figure 3. Adsorption isotherms of uranium ion on porous materials used in this work. $m_{\text {adsorbent }}=10$ $\mathrm{mg}, \mathrm{pH}=4, \mathrm{t}=5 \mathrm{~h}$ and $\mathrm{T}=298 \mathrm{~K}$.

The photo-switching performance towards $\mathrm{U}^{\mathrm{VI}}$ ions in aqueous solution was also explored. Note that samples A-3: 11.7wt\% does not show any photo-switching behavior towards $\mathrm{UO}_{2}{ }^{2+}$, as evidenced by the adsorption capability of it under ambience or after UV irradiation showing almost no detectable alteration. In contrast, such behavior prevailed in A-3: 20.6wt\%, A-3: 28.2wt\%, and A-3: 35.7wt $\%$, resulting in difference $\left(8.7 \%, 14.3 \%\right.$ and $12.3 \%$, respectively) in $\mathrm{U}^{\mathrm{VI}}$ adsorption capacity with/without UV (Figure 4). Interestingly, this can be well recovered with the efficiency of approximately $100 \%$ by reirradiating the same samples under $250 \mathrm{~nm}$, which mainly due to the unique "open-close double doors" system formed by the reversible photodimerization inside A-3 series. In addition, such photo-switching storage and release of $\mathrm{U}^{\mathrm{VI}}$ ions can be further reflected in the eluted experiments. The adsorbed $\mathrm{U}^{\mathrm{VI}}$ ions in A-3: 28.2wt\% can be easily eluted by $0.1 \mathrm{M} \mathrm{HCl}$ with elution efficiency of $80 \%$, whereas identical samples after UV irradiation $(320 \mathrm{~nm})$, under the same eluting conditions, give only a $60 \%$ elution efficiency, indicative of $20 \% \mathrm{U}^{\mathrm{VI}}$ ions (equal to 70 
$\mathrm{mg} / \mathrm{g}$ ) that were sealed inside sorbent by means of such photo-switching technique. Similarly, reirradiating the same samples under $250 \mathrm{~nm}$ completely rereleases the sealed $20 \% \mathrm{U}^{\mathrm{VI}}$ ions (Figure $5)$.

Seen from the above experimental results, it is clear that the $\mathrm{U}^{\mathrm{VI}}$ adsorption capability of microporous Zn-MOF-74 (A-1) is largely enhanced by 58\% when it is prepared as microporous-mesoporous material (A-2), and this is further magnified by $20 \%$ when it is modified by coumarin, resulting in an overall $\mathrm{U}^{\mathrm{VI}}$ adsorption capability of $360 \mathrm{mg} / \mathrm{g}$. Moreover, the coumarin-modified microporous-mesoporous Zn-MOF-74 (A-3) triggered by UV facilitates to generate an "open-close double door" system in the pore outlets, ${ }^{24}$ which consequently allows for reversible photocontrol over $\mathrm{U}^{\mathrm{VI}}$ ions entering into porous materials. This best photo-switching efficiency is estimated to be $14.3 \%(50 \mathrm{mg} / \mathrm{g})$.

As discussed above, relatively speaking, in this study samples A-3: $28.2 \mathrm{wt} \%$ showing high $\mathrm{U}^{\mathrm{VI}}$ adsorption capability of $350 \mathrm{mg} / \mathrm{g}$ and smart photo-switching storage and release of $\mathrm{U}^{\mathrm{VI}}$ in aqueous solution seems more suitable for $\mathrm{U}^{\mathrm{VI}}$ adsorption. Thus, the $\mathrm{U}^{\mathrm{VI}}$ adsorption and stability of it was also confirmed by IR (Infrared Spectrum), TG, PXRD, SEM (Scanning Electron Microscopy) and EDS (Energy Dispersive Spectroscopy). Among these IR bands, significant variety for $\mathrm{C}=\mathrm{O}$ bond after loading of $\mathrm{U}^{\mathrm{VI}}$ ions on MOFs such as appearance of new peak at $1506 \mathrm{~cm}^{-1}$ and disappearance of peak at $1548 \mathrm{~cm}^{-1}$ strongly suggests the interactions between $\mathrm{C}=\mathrm{O}$ groups of coumarin and $\mathrm{U}^{\mathrm{VI}}$ ions. ${ }^{27-28}$ Moreover, the appearance of new peaks of $918 \mathrm{~cm}^{-1}$ in samples A-3: $\mathbf{2 8 . 2 w t} \%$ after loaded $\mathrm{U}^{\mathrm{VI}}$ ions (namely A-3: 28.2 wt $\% @ \mathbf{U O}_{2}{ }^{2+}$ ), typical of the characteristic stretching bond of $\mathrm{UO}_{2}{ }^{2+}$, clearly indicates the adsorption of $\mathrm{U}^{\mathrm{VI}}$ on $\mathbf{A}-\mathbf{3}: \mathbf{2 8 . 2} \mathbf{w t} \%{ }^{19-20}$ While another new peak at $748 \mathrm{~cm}^{-1}$ is in agreement with the formation of new U-O bond, which, most likely, attributes to the ester group of coumarin (Figure S12) ${ }^{19-20}$ Moreover, in the TG plots (Figure S13), the residual weight percentage after $500^{\circ} \mathrm{C}$ for $\mathbf{A - 3 :} \mathbf{2 8 . 2 w t} \% @ \mathbf{U O}_{2}{ }^{2+}$ is 2.7 times bigger than that observed in A-3: $28.2 \mathrm{wt} \%$, which is ascribed to the loading of $\mathrm{U}^{\mathrm{VI}}$ on $\mathbf{A}-3 \mathbf{3}: \mathbf{2 8 . 2 \mathrm { wt }} \%$ that will generate heavier 
uranium compounds after calcinations. Furthermore, as evidenced by PXRD investigations, the PXRD patterns of A-3: 28.2wt\% approximately match that of its $\mathrm{U}^{\mathrm{VI}}$-loaded phase, indicative of

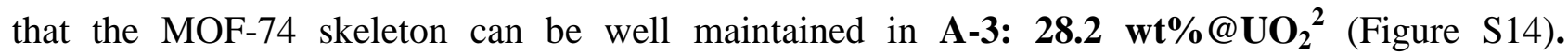
Furthermore, the uptake of $\mathrm{U}^{\mathrm{VI}}$ on A-3: 28.2 wt\% is detected by SEM and EDS analyses (Figure S15, S16). The almost disappear of pore structure in A-3: 28.2 wt\%@ $\mathbf{U} \mathbf{O}_{2}{ }^{2+}$ strongly confirms the loading of $\mathrm{U}^{\mathrm{VI}}$ (Figure S10). The content estimated by EDS is $1: 1$ for $\mathrm{U}: \mathrm{Zn}$, in accordance with the experimental adsorption capability of $350 \mathrm{mg} / \mathrm{g}$.

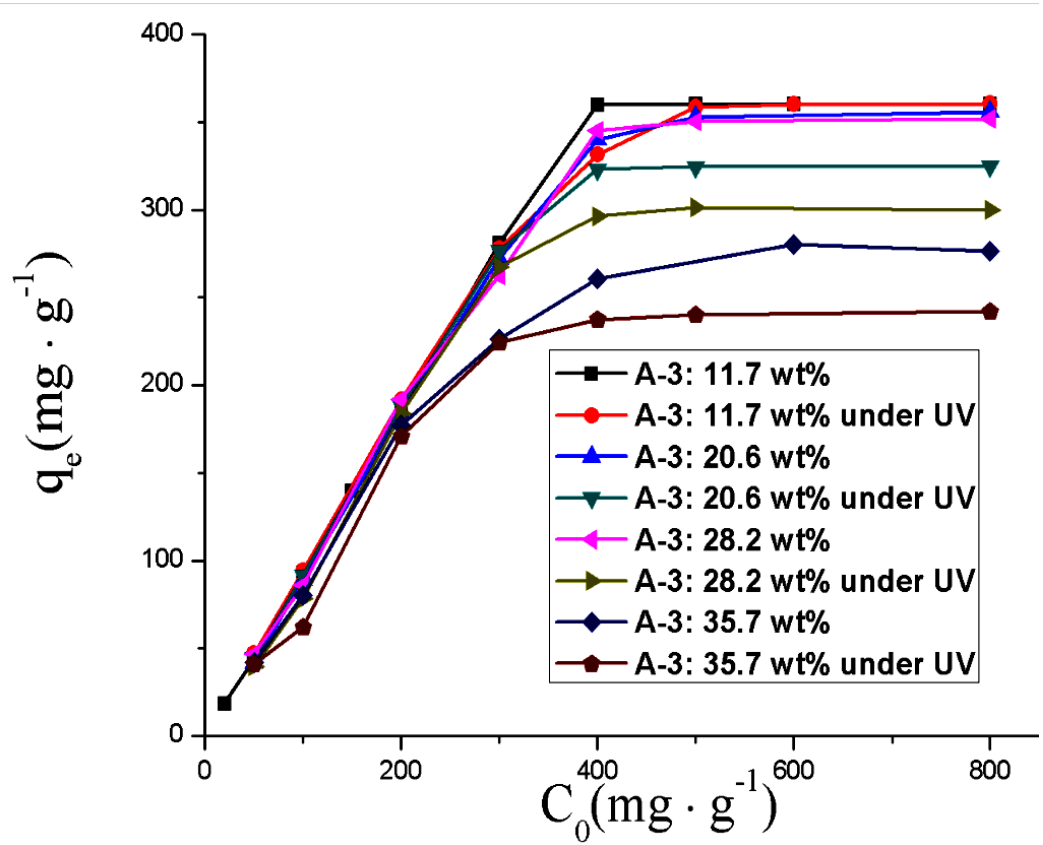

Figure 4. The adsorption isotherms of uranium ion on porous materials under ambience or after UV (320 nm) irradiation. $\mathrm{m}_{\text {adsorbent }}=10 \mathrm{mg}, \mathrm{pH}=4, \mathrm{t}=5 \mathrm{~h}$ and $\mathrm{T}=298 \mathrm{~K}, \mathrm{t}_{\text {irradiation }}=30 \mathrm{~min}$. 


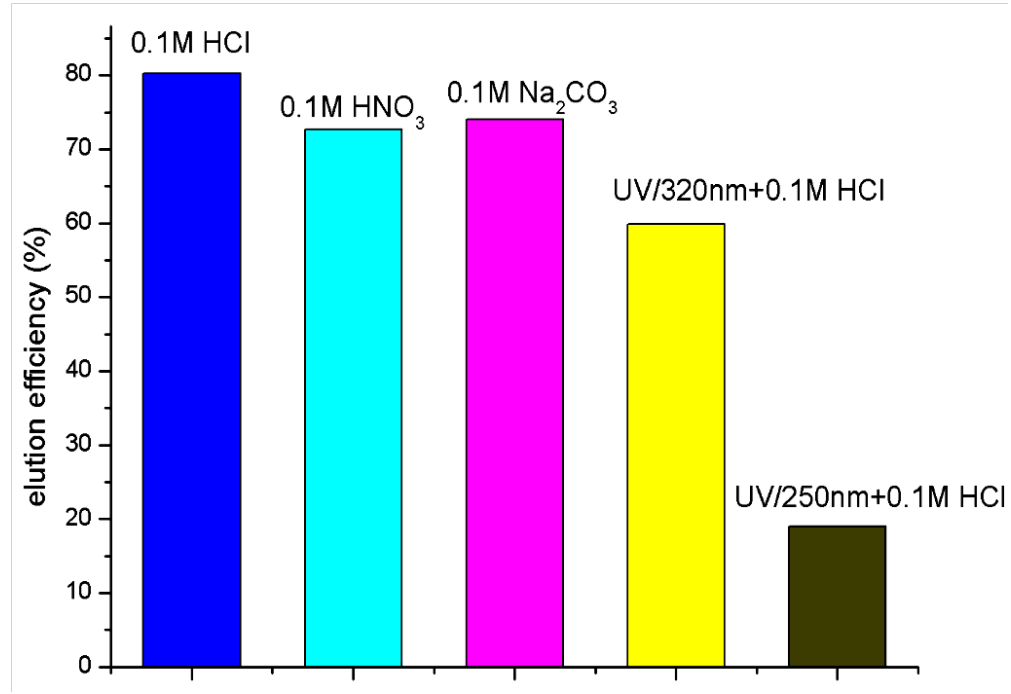

Figure 5. Various elution efficiencies under different eluting conditions for samples A-3: 28.2 wt\% after fully loading $\mathrm{UO}_{2}{ }^{2+}$. Note: the elution colored in yellow is the samples after UV $(320 \mathrm{~nm})$ irradiation for $30 \mathrm{~min}$ and that was eluted by $0.1 \mathrm{M} \mathrm{HCl}$. The elution colored in black represented reirradiating above samples under UV $(250 \mathrm{~nm})$ for $30 \mathrm{~min}$ and then eluting by $0.1 \mathrm{M} \mathrm{HCl}$.

\section{Conclusions}

In summary, we demonstrated here how not only to enhance the $\mathrm{U}^{\mathrm{VI}}$ adsorption capability of MOF materials, but also how to access photo-switching storage and release of $\mathrm{U}^{\mathrm{VI}}$, using simple and economic MOF materials. Selecting Zn-MOF-74, we found that such material in the microporous-mesoporous form could largely enhance the $\mathrm{U}^{\mathrm{VI}}$ adsorption capability by $58 \%$, then doping coumarin inside this material further improved the adsorption performance by $20 \%$. Therefore our work showed an innovatory, simple, and effective pathway to prepare MOF or hybrid MOF materials with high $\mathrm{U}^{\mathrm{VI}}$ adsorption capability. Moreover, based on the reversible photodimerization between two coumarin molecules, the construction of a smart "open-close double door" system on the outlet of MOF materials creates a $14.3 \%$ photo-switching capability for storage/release of $\mathrm{U}^{\mathrm{VI}}$ in aqueous solution at $\mathrm{pH}=4$. Therefore our work here opens up a unique method for green storage/release of $\mathrm{U}^{\mathrm{VI}}$ in aqueous solution. Furthermore, it is known that established photo-switching behavior towards $\mathrm{CO}_{2}$, dye, and fluorescence in MOFs is mainly 
derived from trans-to-cis transformation of azobenzene units or open-ring-to-close-ring transformation of diarylethene unit. ${ }^{29-31}$ By contrast, our work has opened up a gate to access photo-switching behavior towards radioactive $\mathrm{U}^{\mathrm{VI}}$ ion, by means of reversible photodimerization of coumarin molecules.

\section{Acknowledgements}

This work was supported by the Foundation of Key Laboratory for Radioactive Geology and Exploration Technology, Fundamental Science for National Defense and State Key Laboratory for Nuclear Resources and Environment (Z1502), and the NSF of China (21203022, 21261001, 21361001), the Natural Science Foundation of Jiangxi Province of China (20143ACB20002, 20151BAB203001), the Innovation Fund Designated for Graduate Students of Jiangxi Province (YC2015-S274), and the Young scientist training program of Jiangxi Province of China (no. 20142BCB23018).

\section{References}

[1] Nuclear Safety Review for the Year 2010; International Atomic Energy Agency: Vienna, Austria, (2011).

[2] E. S. Craft, A. W. Abu-Qare, M. M. Flaherty, M. C. Garofolo, H. L. Rincavage, M. B. Abou-Donia, Depleted and natural uranium: chemistry and toxicological effects, J. Toxicol. Environ. Health, Part B 7(2004), 297-317.

[3] A. Favre-Reguillon, G. Lebuzit, J. Foos, A. Guy, M. Draye, M. Lemaire, Selective concentration of uranium from seawater by nanofiltration, Ind. Eng. Chem. Res. 42(2003), 5900-5904.

[4] P. Schmitt, P. D. Beer, M. G. B. Drew, P. D. Sheen, Uranyl binding by a novel bis-calix[4]arene receptor, Tetrahedron Lett. 39(1998), 6383-6386. 
[5] L. Al-Attar, A. J. Dyer, Sorption behaviour of uranium on birnessite, a layered manganese oxide, J. Mater. Chem. 12(2002), 1381-1386.

[6] S. M. Webb, C. C. Fuller, B. M. Tebo, J. R. Bargar, Determination of uranyl incorporation into biogenic manganese oxides using X-ray absorption spectroscopy and scattering, Environ. Sci. Technol. 40(2006), 771-777.

[7] K. J. Cantrell, D. I. Kaplan, T. W. Wietsma, Zero-valent iron for the in situ remediation of selected metals in groundwater, J. Hazard Mater. 42(1995), 201-212.

[8] K. L. Toews, N. G. Smart, C. M. Wai, Complexation and transport of uranyl nitrate in supercritical carbon dioxide with organophosphorus reagents, Radiochimica Acta, 75(1996), 179-184.

[9] Y. H. Yang, S. X. Sun, S. Y. Xue, Z. K. Yang, Y. S. Wang, B. Bao, Extraction of uranium(VI) through reversed micelle by primary amine $\mathrm{N}_{1923}$, J. Radioanal Nucl. Chem. 222(1997), 239-241.

[10] A. M. Starvin, T. P. Rao, Solid phase extractive preconcentration of uranium(VI) onto diarylazobisphenol modified activated carbon, Talanta 63(2004), 225-232.

[11] S. J. Coleman, P. R. Coronado, R. S. Maxwell, J. G. Reynolds, Granulated activated carbon modified with hydrophobic silica aerogel-potential composite materials for the removal of uranium from aqueous solutions, Environ. Sci. Technol. 37(2003), 2286-2290.

[12] A. S. Al-Hobaib, A. A. Al-Suhybani, Removal of uranyl ions from aqueous solutions using barium titanate, J. Radioanal Nucl. Chem. 299(2014), 559-567.

[13] H. I. Ulusoy, S. J. Simsek, Removal of uranyl ions in aquatic mediums by using a new material: Gallocyanine grafted hydrogel, J. Hazard Mater. 254(2013), 397-405.

[14] M. Monier, N. H. Elsayed, Selective extraction of uranyl ions using ion-imprinted chelating microspheres, J. Colloid Interface Sci. 423(2014), 113-122.

[15] B. Li, L. J. Ma, Y. Tian, X. D. Yang, J. Li, C. Y. Bai, X. Y. Yang, S. Zhang, S. J. Li, Y. D. Jin, 
A catechol-like phenolic ligand-functionalized hydrothermal carbon: one-pot synthesis, characterization and sorption behavior toward uranium, J. Hazard. Mater. 271(2014), 271, 41-49.

[16] M. J. Manos, M. G. Kanatzidis, Layered metal sulfides capture uranium from seawater, J. Am. Chem. Soc. 134(2012), 16441-16446.

[17] S. L. Ma, L. Huang, L. J. Ma, Y. Shim, S. M. Islam, P. L. Wang, L. D. Zhao, S. C. Wang, G. Sun, X. J. Yang, M. G. Kanatzidis, Efficient uranium capture by polysulfide/layered double hydroxide composites, J. Am. Chem. Soc. 137(2015), 3670-3677.

[18] M. Carboni, C. W. Abney, S. B. Liu, W. B. Lin, Highly porous and stable metal-organic frameworks for uranium extraction, Chem. Sci., 4(2013), 2396-2402.

[19] W. T. Yang, Z. Q. Bai, W. Q. Shi, L. Y. Yuan, T. Tian, Z. F. Chai, H. Wang, Z. M. Sun, MOF-76: from a luminescent probe to highly efficient $\mathrm{U}^{\mathrm{VI}}$ sorption material, Chem. Commun., 49(2013), 10415-10417.

[20] L. L. Wang, F. Luo, L. L. Dang, J. Q. Li, X. L. Wu, S. J. Liu, M. B. Luo, Ultrafast highperformance extraction of uranium from seawater without pretreatment using anacylamideand carboxyl-functionalized metal-organic framework, J. Mater. Chem. A, 3(2015), 13724-13730.

[21] P. K. Thallapally, B. P. McGrail, D. R. Brown, J. Liu, Progress in adsorption-based $\mathrm{CO}_{2}$ capture by metal-organic frameworks, Chem. Soc. Rev., 41(2012), 2308-2322.

[22] T. H. Bae, J. R. Long, $\mathrm{CO}_{2} / \mathrm{N}_{2}$ separations with mixed-matrix membranes containing $\mathrm{Mg}_{2}$ (dobdc) nanocrystals, Energy Environ. Sci., 6(2013), 3565-3569.

[23] Y. F. Yue, Z. A. Qiao, P. F. Fulvio, A. J. Binder, C. C. Tian, J. H. Chen, K. M. Nelson, X. Zhu, S. Dai, Template-free synthesis of hierarchical porous metal-organic frameworks, J. Am. Chem. Soc. 135(2013), 9572-9575.

[24] N. K. Mal, M. Fujiwara, Y. Tanaka, Photocontrolled reversible release of guest molecules from 
coumarin modified mesoporous silica, Nature, 421(2003), 350-353.

[25] M. A. Gomez, M. J. Hendry, J. Kochinsky, J. Elssilfie-Dogan, S. Paikaray, Mineralogical controls of $\mathrm{Mg}$ and $\mathrm{Al}$ in Uranium mill and tailings facilities: Key Lake, Saskatchewan, Canada. Environmental Science \& Technology, 47(2013), 7883-7891.

[26] J. Robertson, K. Shacklock, R. Frey, M. A. Gomez, J. Elssilfie-Dogan, M. J. Hendry, Modelling the Key Lake Mills Bulk Neutralization Process using a Pilot-Scale Model. Hydrometallurgy, 149(2014), 210-219.

[27] K. E. Lee, M. A. Gomez, S. Elouatik, G. P. Demopoulos, Further understanding of the adsorption of N719 complex on Anatase $\mathrm{TiO}_{2}$ films for DSSC applications using Vibrational spectroscopy and Confocal Raman imaging, Langmuir, 26(2010), 9575-9583.

[28] Y. He, Y. Zhao, Y. Zhao, Photoinduced bending of a coumarin-containing supramolecular polymer, Soft Matter. 5(2009), 308-310.

[29] J. Park, D. Q. Yuan, K. T. Pham, J. R. Li, A. Yakovenko, H. C. Zhou, Reversible alteration of $\mathrm{CO}_{2}$ adsorption upon photochemical or thermal treatment in a metal-organic framework, J. Am. Chem. Soc. 134(2012), 99-102.

[30] N. Yanai, T. Uemura, M. Inoue, R. Matsuda, T. Fukushima, M. Tsujimoto, S. Isoda, S. Kitagawa, Guest-to-host transmission of structural changes for stimuli-responsive adsorption property, J. Am. Chem. Soc. 134(2012), 4501-4504.

[31] F. Luo, C. B. Fan, M. B. Luo, X. L. Wu, Y. Zhu, S. Z. Pu, W. Y. Xu, G. C. Guo, Photoswitching $\mathrm{CO}_{2}$ capture and release in a photochromic diarylethene metal-organic framework, Angew. Chem. Int. Ed. 53(2014), 9298 -9301. 